Revue internationale P.M.E.

Économie et gestion de la petite et moyenne entreprise

Revue

internationale

PME

\title{
La gestion de l'interactivité entreprise/environnement
}

\section{Yves Chappoz}

Volume 4, numéro 3, 1991

URI : https://id.erudit.org/iderudit/1008070ar

DOI : https://doi.org/10.7202/1008070ar

Aller au sommaire du numéro

Éditeur(s)

Presses de l’Université du Québec

ISSN

0776-5436 (imprimé)

1918-9699 (numérique)

Découvrir la revue

Citer cette note

Chappoz, Y. (1991). La gestion de l'interactivité entreprise/environnement. Revue internationale P.M.E., 4(3), 53-75. https://doi.org/10.7202/1008070ar

\section{Résumé de l'article}

Pour la petite entreprise, la gestion de l'interactivité avec son environnement joue un rôle essentiel dans son développement. Cet article montre l'importance déterminante du propriétaire-dirigeant et la fonction originale des réseaux médiateurs d'interactivité au sein de la petite entreprise. L'approche typologique qui repose à la fois sur une grille d'analyse précise et une connaissance empirique du terrain, permet de rendre compte de la diversité des pratiques entrepreneuriales. 


\title{
Notes de recherche
}

\section{La gestion de l'interactivité entreprise/environnement}

\author{
Yves CHAPPOZ* \\ Ondaine-Développement
}

\begin{abstract}
RÉSUMÉ
Pour la petite entreprise, la gestion de l'interactivité avec son environnement joue un rôle essentiel dans son développement. Cet article montre l'importance déterminante du propriétaire-dirigeant et la fonction originale des réseaux médiateurs d'interactivité au sein de la petite entreprise. L'approche typologique qui repose à la fois sur une grille d'analyse précise et une connaissance empirique du terrain, permet de rendre compte de la diversité des pratiques entrepreneuriales.
\end{abstract}

\begin{abstract}
For the small business, the managing of the interactivity with its environment plays an essential role in its development. This article shows the important determining factor of the director-owner and the original function of the Interactive Mediators network at the heart of the small business. The typological approach which is set on the one hand on a precise analysis table, and on the other hand, on empirical knowledge of the field, allows to account for the diverse practises of the entrepreneurs.
\end{abstract}

\section{RESUMEN}

En la pequeña empresa la interrelación con el entorno juega un papel esencial en su desarrolo. Este artículo muestra la importancia determinante del Gerente Propietario y la función que desempeñan las redes como elementos mediadores de dicha interrelación para la Pequeña Empresa. Por otra parte, se ha seguido un enfoque tipológico apoyado en un sistema preciso de análisis y en el conocimiento empirico del terreno, lo que le ha permitido demostrar al autor la diversidad de prácticas empresariales.

* Yves Chappoz, docteur en sciences économiques, dirige un organisme parapublic de développement économique et intervient en DESS de Conseil en développement à l'Université de Saint-Etienne, France .

Adresse : Ondaine-Développement, 82, rue Jean Jaurès, 42700 Firminy, France . 


\section{Introduction}

Le développement de la petite entreprise repose sur une dynamique d'ajustements permanents des quatre piliers de son système de compétitivité : environnement, buts, activités, organisation (Marchesnay, 1986), dont l'entrepreneur assure le pilotage. Dans le cas de la petite entreprise, fortement influencée par son méso-système, la maîtrise de la variable « environnement » est essentielle pour anticiper les menaces et saisir les occasions. Cette maitrise repose sur la gestion par le dirigeant de l'interactivité entreprise/environnement. À partir de l'analyse d'une expérience de terrain, nous démontrerons que la gestion de l'interactivité permet à la petite entreprise de desserrer la contrainte environnementale et de se créer un espace de liberté et qu'en plus, elle offre au dirigeant la possibilité de découvrir de nouvelles occasions d'affaires en captant les ressources stratégiques que recèle son environnement.

Dans un premier temps, la présentation d'une expérience de terrain met en évidence le double enjeu de la gestion de l'interactivité. Elle dépend tout d'abord du profil du dirigeant qui en dicte sa perception et sa pratique, mais également et paradoxalement, de la qualité de l'environnement, notamment de la diversité et de la performance des réseaux qui stimulent cette interactivité. Dans la deuxième partie, une analyse des environnements immédiats en termes d'industries, de filières et de marchés, puis des environnements médiats dans l'optique réseaux de compétences, d'acteurs et de villes (technopôles, pôles de compétences) conduit à préciser la nature et la variété des interactivités dans leurs dimensions opérationnelles, organisationnelles et stratégiques. Les réseaux assurent la logistique informative, fiabilisent les transactions d'interactivité et en réduisent les coûts de gestion. Enfin, l'élaboration et la mise en œuvre d'une typologie de profils d'entrepreneurs révèlent que les dirigeants ont rarement le savoir-faire nécessaire pour accéder rapidement et efficacement à leur environnement. Aussi, l'approche des petites entreprises par les structures interfaces néglige-t-elle trop souvent leurs spécificités. 


\section{Expérimentation de l'interactivité entreprise/environnement}

\subsection{Le contexte économique et institutionnel de l'opération :}

Le destin économique de l'Ondaine ${ }^{1}$, bien qu'il présente des spécificités qui lui sont propres, est étroitement lié à celui de l'agglomération stéphanoise à laquelle il appartient. Le contexte économique de cette agglomération ${ }^{2}$ se caractérise par un lent déclin économique amorcé dès les années 60 avec notamment la décision de fermeture des houillères. Le bassin stéphanois reste à l'écart de la recomposition du tissu industriel français qui se dessine au lendemain de la Deuxième Guerre mondiale et semble même refuser de moderniser et de diversifier son industrie.

Ce déclin relatif, longtemps masqué par la prospérité des « trente glorieuses ", éclate dès les années 70 avec la crise du textile, s'amplifie avec le choc pétrolier des années 1973/1974 et atteint son paroxysme avec l'effondrement du groupe Creusot-Loire en 1984, ce qui lui vaut d'être classé «pôle de conversion ».

En trente ans, le paysage industriel s'est totalement transformé. À un système productif localisé (Pecqueur, 1989) dominé par les grandes entreprises industrielles qui structuraient et rythmaient l'activité d'une myriade de soustraitants, se substitue une mosaique de PME orphelines des grands groupes (Banville, 1983) et souffrant d'une situation globale d'isolement et d'atomisation. L'Ondaine, sous-bassin périphérique intimement intégré à l'agglomération stéphanoise et marqué par les houillères et la sidérurgie, n'échappe pas à ce phénomène.

Pour accompagner la reconversion des bassins de vieilles industries, la région Rhône-Alpes propose une procédure intitulée "contrat de développement économique de bassin " (CDEB), qui vise, selon les termes de la circulaire régionale, à mobiliser les acteurs locaux « autour d'un projet de développement adapté aux potentialités et aux contraintes spécifiques du bassin ». Le projet consiste à proposer un ensemble d'actions destinées à créer un environnement favorable au développement des entreprises.

1. L'Ondaine (63000 habitants et 24000 actifs) est un sous-bassin d'emploi de l'agglomération stéphanoise (439000 habitants et 183000 actifs) situé au sud-ouest de Saint-Étienne, Loire, France.

2. Cette synthèse s'appuie sur les travaux du CRESAL, équipe de recherche associée au CNRS, du CREUSET, centre de recherche économique de l'Université de SaintÉtienne et de l'agence EPURES où l'auteur était responsable des études économiques. 


\subsection{Le contrat de développement économique du bassin Ondaine :}

Pour créer une dynamique nouvelle, le contrat de bassin Ondaine s'inscrit dans une démarche intercommunale et partenariale d'élaboration et de mise en œuvre qui associe, au côté des sept communes de l'Ondaine et des collectivités territoriales (région, département), des acteurs publics (préfecture, directions régionales ou départementales de l'Industrie, du Travail, de l'Équipement, etc.), parapublics (chambres consulaires, agences de développement, organisations patronales, etc.) et privés, principalement les entreprises.

Le CDEB se caractérise par un ensemble cohérent d'actions organisées pour doter le « territoire Ondaine » d'avantages concurrentiels (Laboratoire de technologies nouvelles, Maison de l'Ondaine), et pour stimuler les capacités entrepreneuriales et managériales des dirigeants d'entreprises (actions concertées de développement et de modernisation). Notre propos n'est pas de décrire l'ensemble du contrat, mais de pointer les actions qui s'inscrivent dans l'optique de la gestion de l'interactivité entreprise/environnement ${ }^{3}$, notamment de présenter rapidement le Laboratoire de technologies nouvelles et la Maison de l'Ondaine.

La réalisation du Laboratoire de technologies nouvelles, qui entre dans sa phase de fonctionnement, reflète le double souci d'aider les lycées à construire de nouvelles relations avec leur environnement industriel et de requalifier un territoire par la création de pôles de compétences. Ce laboratoire, implanté sur le site d'un lycée technique doté de BTS, vise à consolider la formation initiale et continue, mais également à développer une pratique de transfert de technologies déjà ancienne dans l'établissement vers les moyennes et petites entreprises, dans les domaines de la productique et des automatismes.

L'originalité de la démarche réside dans le recrutement d'un ingénieurconseil venant du monde industriel et chargé de mettre en synergie le lycée, le laboratoire et les entreprises utilisatrices. La philosophie du projet est de mettre à la disposition des entreprises une personne capable de les aider à formuler leurs besoins en ressources technologiques, puis de les accompagner pour réussir la greffe technologique qui passe notamment par la formation des hommes.

3. Le contrat de bassin Ondaine, signé pour une période de 3 ans (1990 à 1992), repose sur des actions qui visent à :

- développer les entreprises existantes ou en accueillir de nouvelles : ACDM, création d'un service commun technico-commercial, mise en place d'une Maison de l'Ondaine.

- enrichir les liens entre l'industrie et les établissements scolaires : Laboratoire de technologie nouvelles, Centre multimédia, Atelier agro-alimentaire.

- transformer et promouvoir l'image de marque de l'Ondaine : événements médiatiques, supports de communication, salons spécialisés. 
La Maison des entreprises de l'Ondaine sera un lieu physique où les entreprises pourront se réunir, se rencontrer, exposer leurs produits et leurs savoir-faire. Par delà l'effet vitrine et la fonction "point de rencontre », la Maison de l'Ondaine, par l'équipe qui l'animera, sera pour les entreprises un centre d'accès aux ressources stratégiques. Autrement dit, sa mission essentielle sera d'éveiller les entreprises aux enjeux de l'environnement, de les inciter à l'activité de veille en leur apportant des informations pertinentes, de les aider à nouer les contacts nécessaires à la captation de ressources stratégiques, bref, de les aider à se créer des réseaux utiles et efficaces. Pour atteindre cet objectif, la Maison de l'Ondaine, qui disposera volontairement de moyens humains réduits, mobilisera les compétences et les savoir-faire des nombreux organismes stéphanois et rhône-alpins, afin de les rendre accessibles aux entreprises ondinoises. La démarche n'a pas pour but de rajouter un organisme supplémentaire aux nombreuses structures existantes, mais de créer un outil de médiation de l'interactivité. La réalisation d'actions concertées de développement et de modemisation et, plus récemment, la création d'un club des entrepreneurs de l'Ondaine, préfigurent la mise en place prochaine de cet outil.

\subsection{Le cas des actions concertées de développement et de modernisation}

\subsubsection{Le projet initial}

Les actions concertées de développement et de modernisation ont été conçues lors de la préparation du contrat de développement économique de l'Ondaine dans le cadre d'un groupe de travail composé de la Chambre de commerce et d'industrie (CCI), de la Chambre de métiers (CdM), du Syndicat métallurgique patronal de la Loire (SMPL), de la Direction régionale de l'Industrie et de la Recherche (DRIR) et de l'agence d'urbanisme EPURES, ce dernier organisme étant chargé de l'élaboration du contrat de bassin.

Partant du double constat que les entreprises qui utilisent les aides au conseil améliorent leurs performances industrielles et commerciales, et que paradoxalement les PE (moins de 50 salariés) saisissent rarement ce type d'opportunités ou en ont une perception négative, le groupe de travail a bâti un projet intitulé " actions concertées de développement et de modernisation " (ACDM). Ce projet, qui cible prioritairement les entreprises de moins de trente personnes, a retenu l'intérêt des organismes tutélaires qui ont accepté de cofinancer l'opération avec les entreprises.

La philosophie du projet consiste à approcher, sur un territoire délimité, le plus grand nombre d'entreprises pour les inciter à réfléchir sur leur situation 
actuelle et future en s'appuyant sur les procédures d'aide au conseil ${ }^{4}$. L'originalité de la démarche repose sur le mixage des suivis collectif et individuel ; suivi collectif par le biais de séminaires pour faciliter le transfert de savoir et de savoir-faire, permettre la confrontation d'idées entre industriels et susciter l'émergence de projets de développement ; suivi individuel par l'apport de prestations personnalisées pour aider le dirigeant à mettre en œuvre ses projets au sein de son entreprise.

Cette action territorialisée propose aux entreprises, compte tenu des caractéristiques industrielles de la cible, une prestation intégrée d'ouverture à leur environnement dans quatre domaines :

- productique-méthode de production,

- stratégie de développement,

- stratégie d'innovation,

- qualité-compétitivité.

L'opération, coordonnée par Ondaine-Développement, dissocie le volet industrie, conduit par la CCI, et le SMPL du volet artisanat animé par la CdM. Elle se déroule en trois étapes parallèles : 1) visites d'incitation, 2) diagnostics d'orientation, 3) actions concertées proprement dites.

Les visites d'incitation reposent sur un entretien d'une heure trente environ. Les consultants mobilisés utilisent un même canevas d'entretien pour identifier l'entreprise et vendre une ou deux des étapes suivantes.

Le diagnostic d'orientation entend faire un rapide examen de l'entreprise pour pointer ses atouts, repérer ses faiblesses et convaincre le dirigeant de franchir l'étape suivante. Le volet artisanat diffère légèrement du volet industrie et propose, lors de cette deuxième étape, des stages de formation au lieu des diagnostics d'orientation.

Les actions concertées reposent, comme nous l'avons déjà dit, sur la constitution de groupes thématiques qui conjuguent les suivis collectif et individuel. Elles se déroulent en trois séquences.

La première séquence de perfectionnement méthodologique consiste à apporter aux dirigeants les outils et les méthodes susceptibles d'aider à l'analyse préalable et la démarche complète d'élaboration d'un projet dans l'un des quatre domaines précités.

4. Les aides au conseil visent à inciter les PME industrielles (moins de 500 personnes) à utiliser les services de consultants spécialisés en couvrant la moitié de la dépense dans la limite d'un plafond variable. 
La seconde séquence, interne à l'organisation, permet à l'entreprise d'élaborer une stratégie, des projets d'actions et un programme de réalisation (objectifs, moyens humains, tèchniques et financiers, échéancier, indicateurs de suivi, etc.).

La dernière séquence de bilan-évaluation consiste essentiellement en une confrontation des expériences conduites par les entreprises associées à l'opération.

\subsubsection{L'épreuve de la réalité}

Sans procéder à une véritable évaluation de l'action ${ }^{5}$ qui entre dans sa phase terminale, il est important de préciser l'état d'avancement de l'opération, son impact et ses difficultés de mise en œuvre.

Le volet industrie qui prévoyait la réalisation d'une centaine de visites d'incitation, d'une trentaine de diagnostics d'orientation et l'implication de 24 entreprises dans des actions concertées peut être considéré comme achevé. Si l'objectif des visites est atteint, actuellement, un seul diagnostic est engagé et huit entreprises sont associées à des actions groupées.

Le volet artisanat, qui prévoyait également la réalisation d'une centaine de visites d'incitation, l'implication d'une cinquantaine d'entreprises à des stages faisant fonction de sas d'orientation et l'association d'une quarantaine d'entreprises à des actions concertées courtes, est à mi-parcours. $\mathrm{La} \mathrm{CdM}$, pour qui cette démarche est totalement nouvelle, a effectué une première cinquantaine de visites sur une courte période, tout en mobilisant un petit nombre de consultants. Cette méthode de travail lui a permis d'être à l'écoute de ses interlocuteurs et d'adapter sa démarche aux attentes ressenties. Après une série de visites-tests auprès d'entreprises artisanales d'activités diverses, la $\mathrm{CdM}$ a focalisé ses visites sur les entreprises sous-traitantes de la mécanique et a abandonné les étapes " formation/diagnostic " et " actions concertées » pour leur substituer un diagnostic de compétitivité (équivalent 3 jours) confié à un consultant sélectionné sur appel d'offre. À ce jour, sur les 27 entreprises qui se sont dites intéressées par le diagnostic de compétitivité lors des visites, seulement 9 d'entre elles se sont réellement engagées dans cette démarche.

\subsubsection{L'intérêt de l'expérimentation}

Ces résultats contrastés invitent à réfléchir sur l'impact de l'opération et l'intérêt de l'expérimentation. Ses enseignements se rapportent à la conception et à la conduite de l'opération, mais également à ce qu'elle révèle sur les petites entreprises et leur gestion de l'interactivité.

5. Notre position de coéquipier au titre de «Ondaine-Développement », ne nous donne pas le recul et la sérénité nécessaires à ces exercices. 
Pour concevoir les ACDM qui ciblent une population hétérogène d'entreprises de petite taille localisées sur un territoire restreint, le groupe de travail a transposé l'expertise et le savoir-faire acquis par la CCI et le SMPL auprès des PME du sud de la Loire. Il a donc donné à l'opération une dimension, une forme et un contenu qui, à l'épreuve du terrain, se sont révélés peu adaptés aux besoins des petites entreprises.

Cette façon d'élaborer l'opération s'est traduite par des objectifs quantitatifs et qualitatifs trop ambitieux. En effet, il s'avère très difficile d'atteindre le taux de participation fixé en contactant une population d'entreprises non sélectionnées ; de plus, cette prestation complète et intégrée ne pouvait intéresser que des entreprises déjà fortement sensibilisées aux enjeux de leur environnement.

Le clivage industrie/artisanat se révèle totalement inadapté et traduit plus les espaces de légitimité institutionnelle des acteurs impliqués, renforcés par la logique financière des procédures administratives utilisées, que la réalité de l'entreprise.

La véritable ligne de partage semble plutôt être la taille de l'entreprise, bien que ce seuil varie selon le type d'activité. Ici, le concept de «petite entreprise » exprime sa pertinence et son opérationnalité.

\section{La petite entreprise face à son environnement}

\subsection{Les spécificités de la petite entreprise}

La PE (moins de 50 salariés) ou la TPE (moins de 10 salariés) se caractérise plus par sa direction personnalisée et son activité spécialisée que par sa taille.

Le propriétaire-dirigeant (PD), parce qu'il est à la fois entrepreneur, manageur et organisateur, personnalise la direction de l'entreprise et polarise son organisation. Le caractère interpersonnel des relations internes et externes caractérise les processus d'information, de négociation et de décision. L'organisation de la production se singularise par une faible spécialisation des tâches et la polyvalence des moyens de production.

L'activité spécialisée repose sur le métier du PD et les missions qu'il assigne à son organisation. Détenteur (ou codétenteur) du savoir-faire, le PD en gère sa transmission, son enrichissement, son renouvellement, voire sa transformation. De même, il adapte les missions de l'entreprise selon l'évolution de la nature des besoins satisfaits. De plus, la qualité des relations avec les partenaires de l'entreprise et la qualité des prestations fournies au client jouent un rôle essentiel dans la survie de la PE. 
Par delà leur hétérogénéité apparente, deux compétences distinctives, la flexibilité et l'interactivité, caractérisent les PE en tant que telles. Notre hypothèse de travail, fondée sur une connaissance pragmatique de la PE acquise sur le terrain, part du constat que ces deux compétences distinctives précitées, loin d'être constitutives de la PE sont des atouts construits et entretenus quotidiennement. Dans un même mouvement, l'accès à la flexibilité passe par une interactivité consciente, de même que l'intensité et la qualité de l'interactivité augmentent avec l'acquisition d'une plus grande flexibilité. Pour donner à ces concepts indissociables toute leur puissance explicative de la réalité des PE et toutes leurs dimensions operationnelles, une analyse des environnements s'impose.

\subsection{Les caractéristiques de l'environnement}

La PE s'insère dans un environnement dense et complexe. Dans la plupart des cas, elle semble en subir fortement l'influence sans exploiter les avantages qu'il recèle. Nous distinguerons deux niveaux, immédiat et médiat, d'environnement.

\subsubsection{L'environnement immédiat : industrie, filière et concurrence}

Ces trois notions d'industrie, de filière et de concurrence, malgré leur proximité conceptuelle apparente, permettent, comme l'a démontré Michel Marchesnay (1991) à maintes reprises, de pointer les trois enjeux clés de la compétitivité de la $\mathrm{PE}$, les variables à maîtriser et les actions à conduire.

Sous l'angle industrie, la PE peut minimiser le risque de vulnérabilité en faisant évoluer son métier pour maintenir ou améliorer sa position concurrentielle. Dans l'optique « marché », la PE atténue le risque de non compétitivité en adaptant ses missions pour développer son avantage concurrentiel. Du point de vue « filière ", la PE, en améliorant les performances de son activité, renforce son pouvoir de négociation et assouplit la gestion de son interdépendance au sein de la filière.

\subsubsection{L'environnement médiat : réseaux de compétences, d'acteurs et de pôles}

L'environnement médiat est formé de l'ensemble des réseaux plus ou moins structurés et formalisés qui irriguent ou enserrent les entreprises. Pour tenter de démêler l'écheveau, nous distinguerons les réseaux de compétences, d'acteurs et de villes. 
TABLEAU 1

L'environnement immédiat

\begin{tabular}{|c|c|c|c|}
\hline & INDUSTRIE & FILIÈRE & MARCHÉ \\
\hline Enjeux & $\begin{array}{l}\text { Position } \\
\text { concurrentielle }\end{array}$ & $\begin{array}{l}\text { Pouvoir de } \\
\text { négociation }\end{array}$ & $\begin{array}{l}\text { Avantage } \\
\text { concurrentiel }\end{array}$ \\
\hline Risques & Vulnérabilité & Dépendance & Non compétitivité \\
\hline $\begin{array}{l}\text { Variables } \\
\grave{a} \\
\text { maîtriser }\end{array}$ & $\begin{array}{l}\text { Complexité } \\
\text { Accessibilité } \\
\text { Turbulence }\end{array}$ & $\begin{array}{l}\text { Concentration } \\
\text { Substitualité } \\
\text { (amont/aval) } \\
\text { Essentialité }\end{array}$ & Combativité \\
\hline \multirow[t]{2}{*}{$\begin{array}{l}\text { Actions } \\
\grave{a} \\
\text { conduire }\end{array}$} & $\begin{array}{l}\text { Veiller } \\
\text { S'organiser }\end{array}$ & $\begin{array}{l}\text { Éviter la } \\
\text { dépendance }\end{array}$ & $\begin{array}{l}\text { Créer une } \\
\text { compétence } \\
\text { distinctive }\end{array}$ \\
\hline & $\begin{array}{l}\text { Organisation } \\
\text { adaptative } \\
\text { Structure } \\
\text { intégrative et } \\
\text { adhocratique }\end{array}$ & $\begin{array}{l}\text { Gérer la } \\
\text { dépendance } \\
\text { Développer } \\
\text { l'interdépendance }\end{array}$ & $\begin{array}{l}\text { Segmentation } \\
\text { commerciale } \\
\text { stratégique } \\
\text { (coût, différenciation) }\end{array}$ \\
\hline
\end{tabular}

Source : Michel Marchesnay (1991)

Les réseaux de compétences, comme le note Gilles Paché, sont « une forme originale de développement d'un certain nombre de grandes entreprises industrielles [...] qui [...] choisissent de recourir aux compétences de sous-traitants, fournisseurs et prestataires de services, plutôt que de posséder leurs propres outils de production ou de distribution physique » (Paché, 1991). L'enjeu pour la PE est de développer l'interdépendance et la dynamique de la transaction externe (Marchesnay, 1991b) pour se positionner favorablement dans la chaîne de valeur.

L'environnement tutélaire, expertal et partenarial (Marchesnay, 1991) forme le réseau d'acteurs. Le réseau tutélaire (Région, DRIRE, Chambres consulaires) joue un rôle de prescripteur et de faciliteur par les aides spécifiques, notamment les aides au conseil, qu'il apporte aux entreprises.

Le réseau public, parapublic ou privé d'expertises apporte ponctuellement aux entreprises les capacités managériales spécialisées dont elles ont besoin pour leur permettre d'accéder aux " ressources stratégiques » nécessaires à leur développement. Xavier Greffe, qui introduit ce concept de « ressources stratégiques », les regroupent en quatre catégories :

- Les ressources financières, de type capital de risques, destinées à consolider les fonds propres de l'entreprise. En effet, les PE souffrent d'une 
sous-capitalisation qui s'explique à la fois par la frilosité du système financier à intervenir en haut de bilan dans les PE où le capital risque d'être piégé et la méfiance des PD qui craignent de perdre leur indépendance en ouvrant leur capital à des personnes extérieures à la famille.

- Les ressources informatives sur les marchés, les concurrents directs ou potentiels, l'évolution des produits et l'apparition de produits de substitutions, etc.

- Les ressources humaines, à savoir la présence de personnes qualifiées capables de mettre en œuvre des technologies modernes de production, mais également l'existence d'un système localisé de formation capable de requalifier les personnes.

- Les ressources technologiques nécessaires à l'évolution de l'activité de l'entreprise (Greffe, 1989).

TABLEAU 2

\section{L'environnement médiat}

\begin{tabular}{|c|c|c|c|}
\hline & $\begin{array}{l}\text { RÉSEAUX DE } \\
\text { COMPETENCES }\end{array}$ & $\begin{array}{l}\text { RÉSEAUX } \\
\text { D'ACTEURS : } \\
\text { - Tutélaire } \\
\text { - Expertal } \\
\text { - Partenarial }\end{array}$ & $\begin{array}{l}\text { RÉSEAUX } \\
\text { DE VILLES } \\
\text { ET POOLES DE } \\
\text { COMPETENCES } \\
\text { (Région) }\end{array}$ \\
\hline Enjeux & $\begin{array}{l}\text { S'inscrire dans la } \\
\text { chaîne de valeur }\end{array}$ & $\begin{array}{l}\text { Accéder aux } \\
\text { ressources } \\
\text { stratégiques au } \\
\text { moindre coût de } \\
\text { transaction }\end{array}$ & $\begin{array}{l}\text { Légitimité sociale } \\
\text { Potentialités logistiques } \\
\text { matérielles et } \\
\text { immatérielles du } \\
\text { territoire }\end{array}$ \\
\hline Risques & $\begin{array}{l}\text { Exclusion des } \\
\text { transactions externes }\end{array}$ & $\begin{array}{l}\text { Non intégration } \\
\text { institutionnelle }\end{array}$ & $\begin{array}{l}\text { Non intégration } \\
\text { sociale et spatiale }\end{array}$ \\
\hline $\begin{array}{l}\text { Variables } \\
\text { à } \\
\text { maîtriser }\end{array}$ & $\begin{array}{l}\text { Coûts de } \\
\text { transactions }\end{array}$ & $\begin{array}{l}\text { Coûts d'accès aux } \\
\text { réseaux et aux } \\
\text { ressources } \\
\text { stratégiques }\end{array}$ & Communications \\
\hline \multirow[t]{3}{*}{$\begin{array}{l}\text { Actions } \\
\text { à } \\
\text { conduire }\end{array}$} & $\begin{array}{l}\text { «Réseauter » } \\
\text { Contracter (contrat } \\
\text { pluriannuel) }\end{array}$ & $\begin{array}{l}\text { Négocier avec } \\
\text { l'environnement } \\
\text { tutélaire }\end{array}$ & $\begin{array}{l}\text { Communiquer } \\
\text { (communication } \\
\text { institutionnelle) }\end{array}$ \\
\hline & $\begin{array}{l}\text { Qualifier l'organisation } \\
\text { (démarche qualité } \\
\text { et certification) }\end{array}$ & $\begin{array}{l}\text { Consulter } \\
\text { l'environnement } \\
\text { expertal }\end{array}$ & Qualifier les hommes \\
\hline & & $\begin{array}{l}\text { Mobiliser } \\
\text { l'environnement } \\
\text { partenarial }\end{array}$ & \\
\hline
\end{tabular}


Le Laboratoire des technologies nouvelles, en proposant ses services dans le domaine de la formation et du transfert de technologies, se positionne en prestataire de ressources stratégiques humaines et technologiques. La présence d'un ingénieur-conseil lui permet de construire une offre personnalisée et adaptée à chaque entreprise.

L'environnement partenarial est formé pour l'essentiel par les fournisseurs de ressources stratégiques technologiques (CETIM, ITF, CRITT, Technopoles, pôles de compétences, etc.), financières (sociétés de capital de risques, sociétés de conversion, etc.), informatives (ARIST, observatoires locaux, etc.) et humaines (établissements de formations secondaires et supérieures, organismes de formation continue, etc.), ainsi que les structures interfaces (agences de développement, organismes d'industrialisation, etc.) dont les missions consistent à faciliter l'interactivité entreprise/environnement.

Les réseaux de villes et de pôles de compétences d'une région forment l'armature du territoire qui n'est plus perçu comme un espace indifférencié polarisé par des villes hiérarchisées, mais analysé comme un complexe de réseaux interconnectés. D'un point de vue entrepreneurial, l'attractivité du territoire dépend de la diversité des réseaux et de la densité des nœuds du maillage, points d'ancrage des entreprises.

Ce maillage doit être compris dans sa dimension physique : les nœuds de communication où s'implantent les grandes surfaces commerciales, les parcs d'activités « haut de gammes », etc., ainsi que dans sa dimension immatérielle : réseaux de communications téléphoniques, télématique, informatique, etc. Plus globalement, la qualité logistique matérielle et immatérielle d'un territoire est essentielle pour permettre aux réseaux de compétences qui substituent le temps à la distance de se développer ou aux réseaux d'acteurs, d'être ou de se rendre accessibles. Autrement dit, une offre logistique performante permet de fiabiliser et d'intensifier les transactions, et également, d'en réduire les coûts.

Ainsi analysé, le "mix » de réseaux forme le méso-système, ensemble complexe souvent perçu comme inaccessible par les $\mathrm{PE}$, alors que paradoxalement, il constitue son espace de légitimation sociale, de communication institutionnelle et de veille technologique, commerciale et stratégique.

Les PE ne soupçonnent pas ou peu l'interêt d'explorer et d'exploiter les ressources stratégiques de leur environnement, personnalisées par les structures interfaces qui en facilitent l'accès.

\subsection{Nature et variété des interactivités}

L'interactivité et la flexibilité sont des compétences distinctives que la PE se doit d'acquérir pour préserver sa compétitivité et réussir son intégration dans le méso-système auquel elle appartient. Cultiver une grande perméabilité avec ses 
environnements immédiat et médiat, permet à la PE, par le biais des réseaux de compétences, de capter les ressources financières, humaines, technologiques et informatives nécessaires à sa compétitivité.

\subsubsection{Nature des interactivités}

Cette interactivité, étroitement corrélée à la flexibilité, s'exprime en termes opérationnels, organisationnels et stratégiques.

TABLEAU 3

\section{Interactivité}

\begin{tabular}{llll}
\hline & Opérationnelle & Organisationnelle & Stratégique \\
\hline Interactivité & $\begin{array}{l}\text { Intensité et qualité } \\
\text { des échanges entre } \\
\text { les membres de } \\
\text { l'organisation } \\
\text { Vlexibilité }\end{array}$ & $\begin{array}{l}\text { Structure organiciste } \\
\text { et adhocratique }\end{array}$ & $\begin{array}{l}\text { Densité et régularité } \\
\text { des liens avec les } \\
\text { partenaires extérieurs }\end{array}$ \\
& $\begin{array}{l}\text { ment des ressources } \\
\text { humaines, } \\
\text { productives et } \\
\text { technologiques }\end{array}$ & $\begin{array}{l}\text { Culture d'entreprise } \\
\text { fondée sur } \\
\text { l'acceptation de } \\
\text { changer de tâches, } \\
\text { d'activités }\end{array}$ & $\begin{array}{l}\text { Nombre de degrés de } \\
\text { liberté par rapport } \\
\text { au méso-système } \\
\text { stratégique }\end{array}$ \\
& $\begin{array}{l}\text { Surqualification } \\
\text { des hommes }\end{array}$ & $\begin{array}{l}\text { Capacité de } \\
\text { mobilisation } \\
\text { du PD }\end{array}$ & $\begin{array}{l}\text { Aptitude et capacité } \\
\text { du PD à scruter son } \\
\text { environnement, à } \\
\text { saisir les occasions } \\
\text { de développement et }\end{array}$ \\
& $\begin{array}{l}\text { équipements des } \\
\text { productifs } \\
\text { Technologie } \\
\text { en grappe }\end{array}$ & & $\begin{array}{l}\text { à capter les ressources } \\
\text { stratégiques néces- } \\
\text { saires }\end{array}$ \\
\hline
\end{tabular}

Source: Michel Marchesnay (1991)

L'interactivité stratégique se caractérise par la densité, la diversité et la régularité des liens avec les partenaires extérieurs. Sa mise en œuvre repose sur l'aptitude du PD à pratiquer la « communication externe stratégique » (Bartoli, 1990), notamment avec les médiateurs d'interactivité sur sa capacité de veille pour prévoir les événements commerciaux, technologiques, sociaux, etc. qui peuvent influencer l'entreprise, sur son habileté à saisir les occasions et à capter, dans son environnement, les ressources stratégiques nécessaires à son développement. L'interactivité stratégique crée un espace de liberté qui permet à l'entreprise de desserrer les contraintes environnementales et d'améliorer sa capacité d'adaptation, bref, d'atteindre la flexibilité stratégique. 
L'interactivité stratégique repose, sur le plan organisationnel, sur une structure de type organiciste rapidement mobilisable et flexible dans ses activités, et exige, sur le plan opérationnel, entre les membres de l'organisation, des échanges intenses et de qualité qui facilitent l'enrichissement et la variété de l'agencement des ressources humaines, productives et technologiques. Le respect du «mix » interactivité/flexibilité permet au PD de rester en congruence avec ses réseaux, d'améliorer la symbiose avec son milieu et de maximiser la captation des occasions d'affaires et des ressources stratégiques. Une culture d'entreprise fondée sur le changement renforce la dynamique d'interactivité.

\subsubsection{Variété et gestion des interactivités}

En introduisant le concept de ressources stratégiques, Xavier Greffe (1989) identifie les quatre points nodaux de ressources: technologiques, informatives, financières et humaines dans l'interactivité PE/environnement. Sans toutefois en préciser les modalités, il suggère d'aménager l'espace intermédiaire entre le marché et les entreprises en créant des espaces de coopération pour mobiliser ou produire les ressources stratégiques nécessaires aux entreprises du milieu.

Michel Rousseau (1989), à partir de l'analyse d'actions collectives du même type que les ACDM, amorce une réflexion sur la gestion de l'interactivité. Il montre l'intérêt, pour l'entreprise, de mobiliser les compétences environnementales et l'importance, pour la communauté économique, de stimuler et d'accompagner le développement des entreprises d'une même zone d'animation économique en s'appuyant sur un institut départemental de management.

Dans le prolongement des travaux de Michel Marchesnay, les concepts d'environnement tutélaire, expertal et partenarial (qui sont autant de réseaux spécifiques, mais souvent étroitement imbriqués) permettent d'analyser plus finement les conditions et les modalités de l'interactivité environnement/ entreprise.

Les réseaux tutélaires jouent un rôle de prescripteur et, en s'appuyant notamment sur la gestion des FRAC (fonds régional d'aide au conseil), incitent (financièrement) les entreprises à utiliser les ressources de leur environnement expertal et partenarial. Le réseau expertal apporte les connaissances et le savoirfaire nécessaires à la mise en œuvre d'une nouvelle organisation, à l'introduction d'une nouvelle technologie, etc. Après avoir préparé le terrain, l'expert aidera l'entreprise à mobiliser le réseau partenarial détenteur de ressources stratégiques, c'est-à-dire qu'il aidera l'entreprise à trouver l'organisme capable d'assurer la prestation de formation adaptée à ses besoins spécifiques ou le centre de recherche apte à l'accompagner dans son effort de $\mathrm{R}$ - D. La ligne de partage entre prescripteurs/experts/partenaires est souvent floue et l'entreprise cliente discerne difficilement où commence et se termine la mission de chaque organisme ou prestataire d'autant que certains cumulent plusieurs de ces missions ; 
ou bien, prisonniers d'une coopération rodée, prescripteurs/experts/partenaires se limitent à un petit nombre d'acteurs au risque de ne plus apporter aux entreprises les prestations adaptées à la réalité de leur situation.

Notre expérience professionnelle nous conduit à mettre en évidence l'émergence d'un réseau de médiateurs d'interactivité, composé de structures d'interfaces spécialisées ou généralistes. Leur but est d'aider les entreprises, à partir d'un diagnostic stratégique, à créer les connections pertinentes avec leur environnement tutélaire, expertal et partenarial pour leur faciliter l'accès aux ressources stratégiques spécifiques ou à spécifier. Leur mission consiste essentiellement à réduire et à fiabiliser les coûts de transaction d'interactivité, autrement dit, à apprendre ou à aider les entreprises à créer des réseaux, avec intelligence et efficacité.

Plus globalement, cette analyse fonde la pertinence d'opérations collectives sur le besoin d'apprendre aux PD à percevoir, écouter et pratiquer leur environnement et, dans certain cas, à prolonger l'action par la création d'interfaces légères. Mais la prise en compte des spécificités de la PE et principalement du profil du PD améliore l'efficacité et l'efficience de ce type d'opération.

\section{Profil du dirigeant-propriétaire et attitudes vis-à-vis de l'environnement}

Le personnage clé de l'interactivité entreprise/environnement est le PD. La décision de «subir » son environnement, c'est-à-dire de le vivre tel qu'il se présente à lui ou de "l'investir » pour dépasser le champ des contacts immédiats et l'explorer dans ses formes médiates, lui revient. Ce travail d'investigation, orienté vers la recherche d'occasions d'affaires ou de captation de ressources stratégiques, le PD peut le faire seul ou en s'appuyant sur les réseaux tutélaire, expertal, partenarial et de médiateurs d'interactivités, mais sa pratique dépendra essentiellement de son profil, nuancé par les variables activité et organisation.

\subsection{Typologie des profils d'entrepreneurs :}

La typologie - PIC (Pérennité, Indépendance, Croissance) et CAP (Croissance, Autonomie, Pérennité) - proposée par Michel Marchesnay, laquelle s'appuie sur les travaux d'investigations et de recherches menés par l'ERFI ${ }^{6}$ et le GREPME ${ }^{7}$, se révèle opérante sur le terrain, à la fois pour comprendre comment fonctionne l'interactivité et pour agir sur son enrichissement. Toutefois, cette typologie doit être utilisée comme un outil heuristique et non comme une catégorisation normative. 
La combinaison des déterminants clés des buts du PD (pérennité, indépendance ou autonomie, croissance) conduit à opposer le PIC au CAP. Le PIC adopte un comportement fortement influencé par une démarche patrimoniale et, dans son environnement, sera sensible aux éléments de stabilité qui pérennisent son affaire et préservent son indépendance, au risque, en forçant le trait, de végéter. Diamétralement opposé, le CAP, motivé par une démarche et une culture entrepreneuriale, aura un comportement du type opportuniste qui le conduira, tout en conservant une autonomie relative, à privilégier la croissance au risque extrême de menacer l'existence même de son affaire.

À partir de ces types théoriques qui se rencontrent rarement à l'état pur, nous proposons quatre types de profils de PD : PIC-artisan, PIC-réactif, PICproactif ou CAP-proactif et CAP-anticipatif ou opportuniste.

a) Le PIC-artisan a une démarche patrimoniale limitée par une recherche de pérennité fondée sur l'indépendance financière à laquelle il sacrifie la croissance de son affaire. Technicien dans l'âme, il adopte une attitude d'évitement vis-à-vis de son environnement qui se traduit par l'absence de démarche commerciale et par voie de conséquence, d'interactivité.

b) Le PIC-réactif, sensible à son indépendance financière, opte pour la croissance sans risquer sa pérennité. Technicien attentif à sa clientèle, il tend à privilégier le service apporté. Son attitude adaptative, même si son interactivité se limite à sa clientèle, lui permet de rester en phase, en termes d'activités et de missions, avec son environnement immédiat.

c) Le PIC-proactif ou le CAP-proactif privilégie la croissance tout en préservant son indépendance ou son autonomie. Dans ce dernier cas, il accepte d'ouvrir son capital à des partenaires neutres, telles que les sociétés régionales de capital de risques. Son attitude composite et adaptative/ opportuniste le conduit à privilégier les relations commerciales de type partenariales, et à développer l'interactivité avec ses différentes formes d'environnement, notamment les réseaux de compétences.

d) Le CAP-anticipatif ou opportuniste recherche la puissance pour occuper dans son industrie une position concurrentielle forte, pour se doter, dans sa filière, d'un réel pouvoir de négociation et acquérir sur son marché un avantage compétitif fondé sur l'offre d'une "fonction» répondant aux exigences de certification les plus rigoureuses. Sa tendance stratégique est de se positionner en entreprise pivot d'un réseau de compétences.

6. Équipe de Recherche sur la Firme et l'Industrie, Université de Montpellier I.

7. Groupe de recherche en économie et gestion des petites et moyennes entreprises, Université du Québec à Trois-Rivières. 
TABLEAU 4

Typologie des profils d'entrepreneurs

\begin{tabular}{|c|c|c|c|c|c|}
\hline $\begin{array}{l}\text { Types de proprié- } \\
\text { taires dirigeants }\end{array}$ & Buts & $\begin{array}{l}\text { Activités ou } \\
\text { Missions }\end{array}$ & Organisation & Environnement & $\begin{array}{l}\text { Tendance } \\
\text { stratégique }\end{array}$ \\
\hline PIC-artisan & $\begin{array}{l}\text { PÉRENNITÉ } \\
\text { INDÉPENDANCE } \\
\text { CROISSANCE }\end{array}$ & $\begin{array}{l}\text { Produire un bien } \\
\text { Technicité }\end{array}$ & $\begin{array}{l}\text { Inexistante } \\
\text { Polarisée par le PD }\end{array}$ & $\begin{array}{l}\text { ÉVTTER } \\
\text { " sans les clients tout } \\
\text { serait pour le mieux } \\
\text { sans parler des } \\
\text { administrations ! " }\end{array}$ & $\begin{array}{l}\text { Absence de stratégie } \\
\text { Interactivité et } \\
\text { flexibilité faibles } \\
\text { ou nulles }\end{array}$ \\
\hline PIC-réactif & $\begin{array}{l}\text { PÉRENNITÉ } \\
\text { INDÉPENDANCE } \\
\text { CROISSANCE }\end{array}$ & $\begin{array}{l}\text { Apporter un } \\
\text { « service " } \\
\text { support : } \\
\text { bien matériel }\end{array}$ & $\begin{array}{l}\text { Légère } \\
\text { De type organiciste }\end{array}$ & $\begin{array}{l}\text { S'ADAPTER : } \\
\text { attitude réaliste } \\
\text { " les choses étant } \\
\text { ce qu'elles sont, } \\
\text { on fait avec " }\end{array}$ & $\begin{array}{l}\text { Stratégie adaptative : } \\
\text { fidéliser la clientèle } \\
\text { Avantage compétitif } \\
\text { de type « qualité- } \\
\text { délais-prix » } \\
\text { Acquérir de l'interacti- } \\
\text { vité et de la flexibilité }\end{array}$ \\
\hline $\begin{array}{l}\text { PIC-proactif ou } \\
\text { CAP-proactif }\end{array}$ & $\begin{array}{l}\text { CROISSANCE } \\
\text { INDÉPENDANCE } \\
\text { OU AUTONOMIE } \\
\text { PÉRENNITÉ }\end{array}$ & $\begin{array}{l}\text { Etre partenaire } \\
\text { support : bien } \\
\text { enrichi d'un service }\end{array}$ & $\begin{array}{l}\text { De type organiciste } \\
\text { avec un embryon de } \\
\text { structure }\end{array}$ & $\begin{array}{l}\text { PRÉCÉDER } \\
\text { « garder une petite } \\
\text { longueur d'avance } \\
\text { sur les copains " }\end{array}$ & $\begin{array}{l}\text { Stratégie composite } \\
\text { adaptative/opportu- } \\
\text { niste : stabiliser la } \\
\text { relation commerciale } \\
\text { Avantage compétitif } \\
\text { de type \& Juste-à-temps » } \\
\text { Développer l'inter- } \\
\text { activité et la flexibilité } \\
\text { acquises }\end{array}$ \\
\hline $\begin{array}{l}\text { CAP-anticipatif } \\
\text { ou opportuniste }\end{array}$ & $\begin{array}{l}\text { CROISSANCE OU } \\
\text { PUISSANCE } \\
\text { AUTONOMIE } \\
\text { PÉRENNITÉ }\end{array}$ & $\begin{array}{l}\text { S'inscrire dans un } \\
\text { réseau de } \\
\text { compétences } \\
\text { support : fonction } \\
\text { (ou bien) enrichie } \\
\text { d'un service }\end{array}$ & $\begin{array}{l}\text { De type organiciste, } \\
\text { mais avec des } \\
\text { risques de rigidité } \\
\text { liés à la structuration } \\
\text { de l'organisation }\end{array}$ & $\begin{array}{l}\text { ANTICIPER } \\
\text { VISION } \\
\text { L'environnement est } \\
\text { un champ à explorer } \\
\text { et à exploiter. } \\
\text { « conjuguons } \\
\text { nos talents " }\end{array}$ & $\begin{array}{l}\text { Stratégie opportuniste: } \\
\text { capter la « désintégra- } \\
\text { tion contrôlée » des } \\
\text { grands groupes ou } \\
\text { entreprises } \\
\text { Avantage compétitif } \\
\text { de type « réduction et } \\
\text { fiabilisation des coûts } \\
\text { de transaction» } \\
\text { Préserver et systéma- } \\
\text { tiser l'interactivité et } \\
\text { la flexibilité acquises }\end{array}$ \\
\hline
\end{tabular}




\subsection{Mise en œuvre de la typologie}

Pour tester l'opérationnalité de cette typologie, nous étudierons le cas de quatre entreprises $^{8}$ de sous-traitance de la mécanique. Ce secteur se caractérise par une vulnérabilité liée à sa forte accessibilité, aux turbulences technologiques (nouveaux matériaux, automatisation), organisationnelles (juste-à-temps, qualité) et commerciales (évolution des exigences des acheteurs). Les grands donneurs d'ordres dominent la filière sur un marché agressif en période de basse conjoncture.

Les dirigeants (un tandem père-fils) de MECA1 (7 personnes) forment l'archétype du PIC-artisan. Cette entreprise travaille exclusivement en soustraitance d'usinage de pièces mécaniques tournées de grandes dimensions, unitaires ou en très petites séries et dispose d'un bel outil de production implanté dans des locaux neufs et fonctionnels. Malgré cette apparence de modernité, les $\mathrm{PD}$ privilégient la pérennité au détriment de la croissance et de la diversification de leur portefeuille de clients, dont l'un des donneurs d'ordres représente plus de $50 \%$ du chiffre d'affaires. Pour gérer leur dépendance vis-à-vis de leurs quatre principaux clients, les PD ont su nouer et entretenir des relations personnalisées avec leurs donneurs d'ordres fondées sur les délais et la disponibilité, doublées d'une capacité de dépannage.

MECA1, dont l'activité repose sur un savoir-faire banalisé, souffre d'une organisation inexistante polarisée sur les PD qui assument l'ensemble des fonctions de direction et de gestion et qui confisquent l'acquisition de nouveaux savoir-faire tel que l'apprentissage de la conduite du tour à commande numérique. Cette attitude se traduit par un manque de disponibilité des PD et une écoute qualitative insuffisante de la clientèle, laquelle tend à reprocher à l'entreprise un retard relatif dans le domaine du contrôle qualité. Cette interactivité insuffisante avec sa clientèle et son environnement, ce dont l'entreprise commence à prendre conscience, induit un risque de décalage entre les missions assignées par les PD et les attentes des principaux donneurs d'ordres.

MECA2, qui fabrique également des pièces unitaires ou en petites séries, construit des ensembles complexes (de type machines spéciales) sur les spécifications du client ou procède à des opérations de maintenance ou de dépannage, ressemble et s'oppose à la fois à MECA1.

Leurs points communs sont leurs tailles (7 personnes), leurs activités qui reposent sur un savoir-faire banalisé, leurs outils de production conventionnels et leurs fortes situations de dépendance. MECA2 réalise $80 \%$ de son chiffre d'affaires avec 5 grandes entreprises métallurgiques de la région.

8. MECA1, MECA2 et MECA3 ont fait l'objet, dans le cadre du CDEB Ondaine, d'un diagnostic de compétitivité réalisé par Alain Fayolle du cabinet de conseil en management STRATEQUIP. 
Mais MECA2 se distingue par son organisation de type organisciste. Le PD s'appuie sur un chef d'atelier et une secrétaire administrative, tous deux détenteurs de parts dans la SARL, pour être disponible à sa clientèle et à son environnement. Cette attitude d'ouverture interactive permet au PD, un PICréactif, d'ajuster ses missions aux évolutions des attentes de sa clientèle. MECA2 développe un service complet de maintenance et de dépannage fondé sur l'écoute, la disponibilité et la rapidité d'action où le service l'emporte sur la fabrication des pièces de remplacement, même si deux de ses clients notent quelques points faibles dans le dispositif qualité. Réactif aiguillonné par ses clients, le PD opte pour une stratégie adaptative de gestion de la dépendance fondée sur une démarche qualité active qui implique l'ensemble du personnel et une action commerciale basée sur la formation et la prospection. Cette action commerciale est complétée par une ouverture progressive (utilisation des aides au conseil) à son environnement immédiat.

MECA3 (20 personnes) est une entreprise de mécanique générale spécialisée dans la fabrication de petites pièces de haute précision en métaux non ferreux. Cette entreprise, dont l'activité repose sur un savoir-faire moins banalisé, dispose d'un outil de production performant dont l'agencement, reflet d'une croissance faite d'occasions saisies au coup par coup, souffre d'un manque de rationalité.

En effet, le PD de MECA3, un proactif à forte tendance CAP, a exploité les difficultés de ses voisins pour se structurer et développer son entreprise. Il s'associe avec l'un d'eux, un concurrent direct en quasi-cessation de paiement qui occupe depuis les fonctions de chef d'atelier et embauche un autre voisin dont l'entreprise avait déposé le bilan, comme responsable de la qualité. Ce mode de croissance lui permet de constituer une équipe soudée, puis de se consacrer principalement à la gestion de l'interactivité externe vis-à-vis de ses fournisseurs et de ses clients.

Pour desserrer l'étreinte des fournisseurs, MECA3 propose à ses partenaires-concurrents de constituer une centrale d'achat. Devant leur refus, le PD crée une société de négoce en métaux (2 personnes) qui lui permet de s'approvisionner à meilleur compte et de se positionner en fournisseur de matières vis-à-vis de ses concurrents.

Poussé par deux de ses clients à évoluer vers la sous-traitance fonctionnelle qui consisterait à réaliser des ensembles mécaniques simples de 20 pièces environ, mais freiné dans ses initiatives par son associé, un PIC-artisan, le PD projette de créer une nouvelle société ; mais une conjoncture incertaine le conduit à différer le projet. MECA3, sans anticiper sur les événements, développe donc une stratégie gradualiste, à la fois opportuniste et adaptative, nourrie par une interactivité que le PD apprend empiriquement à élargir à la veille technologique et au réseautage. 
MECA4, dont le PDG, un ingénieur doublé d'une formation de gestionnaire, est le prototype du CAP-anticipatif et visionnaire (Filion, 1991), développe une stratégie opportuniste de captation de la désintégration contrôlée des grandes entreprises. Constatant que la stratégie de recentrage des grandes entreprises sur leur métier s'accompagne d'un double mouvement de transfert à leurs sous-traitants d'activités dites non stratégiques et d'un resserrement des liens de partenariat avec un nombre réduit de sous-traitants capables d'apporter un ensemble de prestations intégrées, le PD développe une « vision » d'entreprise pivot et conçoit une stratégie de croissance partenariale ciblée qu'il met en œuvre avec l'appui de grands groupes, tel qu'Usinor-Sacilor, qui cherchent à désinvestir au moindre coût industriel et social. Ces derniers, par des accords commerciaux pluriannuels et avec le concours financier de sociétés de conversion (SOFIREM, SODICEM) et des sociétés régionales de capital de risques, sécurisent la reprise d'activité par MECA4 qui fournit à ces mêmes sociétés des prestations de sous-traitance de haut niveau, consolidées par des capacités d'études, de montage et de maintenance.

En six ans, MECA4 est devenu un groupe industriel (330 personnes) composé d'un ensemble d'entreprises ou d'établissements complémentaires dont aucun ne compte plus de 120 salariés, structuré autour d'un holding qui concentre les fonctions stragégiques et tend à s'imposer localement comme une entreprise pivot, leader d'un réseau de compétences.

L'application de cette typologie à quatre entreprises du secteur de la mécanique souligne des pratiques différenciées de l'environnement et de sa gestion.

\subsection{Types d'entrepreneurs et gestion de l'Interactivité}

Le profil du dirigeant influence sa perception en profondeur et en extension de son environnement. Ce dernier est perçu comme une contrainte pesante par le PIC-artisan, lequel opte alors pour une attitude d'evitement qui le conduit à limiter les relations externes à l'entreprise à quelques clients et fournisseurs sans chercher à élargir le champ de ses relations.

Le PIC-réactif découvre que l'écoute active de la clientèle permet d'adapter son activité. Interactif avec ses clients, MECA2 colle à l'évolution de leurs besoins et développe les prestations de maintenance-dépannage qui exigent disponibilité, rapidité et fiabilité. Cette inflexion de ses missions conduit le dirigeant à solliciter son environnement tutélaire et expertal pour l'aider à acquérir un savoir-faire commercial et à mettre en place une démarche qualité.

Le PIC-proactif ou le CAP-proactif enrichit son approche de l'environnement par l'apprentissage de la veille et du réseautage. MECA3 tire parti des vérifications qualité réalisés dans son entreprise par ses donneurs d'ordres, pour 
améliorer sa propre démarche qualité ou profite des visites régulières à ses clients pour s'intéresser à l'évolution de leurs produits et de leurs procédés de fabrication, et prendre en compte leurs incidences sur sa propre activité. Mais sa veille technologique et commerciale reste embryonnaire.

Son environnement devrait stimuler ce savoir-faire naissant comme il tente de le faire avec une entreprise (7 personnes) qui fabrique des produits de frottement autolubrifiants. Avec l'appui d'un organisme parapublic qui assure une double mission tutélaire et expertale, le dirigeant envisage, par intégration en amont, de se lancer dans la fabrication de matériaux composites. Ce choix induit l'apprentissage d'un nouveau métier et la mise en place d'une organisation structurée. On peut s'interroger sur l'efficience de la démarche de cet organisme tutélaire qui oriente l'entreprise vers des choix qui bouleversent complètement ses buts, son métier, ses missions et son organisation sans faire au préalable de diagnostic stratégique, des choix qui entrent en contradiction avec la culture entrepreneuriale du PD. Le dirigeant lors d'une négociation avec un revendeur italien qui, pour offrir une gamme complète de produits, tenait absolument à les commercialiser, découvre que son "métier " est d'être un spécialiste incollable du frottement. Autrement dit, à la stratégie de diversification proposée par l'organisme tutélaire, il préfere intuitivement une stratégie de spécialisation qu'il met en place graduellement en apprenant à usiner les plastiques.

Le CAP-anticipatif et opportuniste, plus fréquent dans les moyennes et grandes organisations que dans le monde de la petite entreprise, s'organise et se structure pour systématiser la veille commerciale, sociale, technologique, etc. Le dirigeant tend à conserver l'activité de réseautage. C'est le cas de MECA4 où ces activités sont assurées, au niveau du holding, par un petit noyau de professionnels performants pour préserver la flexibilité et la réactivité de l'équipe. 


\section{Conclusion}

L'interactivité se révèle être un enjeu de gestion stratégique pour la petite et la moyenne entreprise. Un maillage réussi lui permet de vivre en symbiose dans son méso-système et d'y évoluer de façon synchrone. Mais cet enjeu est rarement perçu par le PD qui se sent désarmé pour aborder ses environnements.

La mise en place d'actions concertées et collectives permet de sensibiliser les PD aux enjeux de leurs environnements, mais également, pour les plus réceptifs d'entre eux, de faciliter l'apprentissage des techniques de veille, de réseautage et de communication stratégique.

Toutefois, comme nous venons de le démontrer, pour être pleinement efficace, c'est-à-dire pour qu'elles se traduisent par l'acquisition d'un savoirfaire entrepreneurial et managérial, ces actions devraient être conçues et pilotées à partir de la carte des profils des PD ciblés.

Les réseaux tutélaire et expertal tiennent rarement compte de la diversité des profils des PL et offrent donc des produits standardisés et indifférenciés répondant imparfaitement à leurs besoins. Paradoxalement, les réseaux médiateurs d'interactivité amélioreraient leur efficacité et leur efficience en étant plus finement attentifs aux besoins spécifiques de leurs entreprises clientes.

Cette investigation mérite donc d'être poursuivie notamment dans trois directions :

1) réaliser des études transversales et longitudinales pour élaborer une méthodologie de gestion de l'interactivité. L'analyse comparative d'actions semblables réalisées sur des territoires et dans des contextes différents permettrait de construire une méthode et des outils, de même que le suivi-évaluation d'une action répétée à intervalles réguliers, sur un même territoire, permettrait de valider la pertinence de la démarche.

2) affiner, problématiser, tester et valider la typologie des entrepreneurs qui est la clé d'accès à la gestion de l'interactivité en réalisant notamment des études cliniques.

3) analyser, dans l'optique Sciences de gestion, la stratégie et les piliers de la compétitivité des organismes médiateurs d'interactivité pour déterminer les conditions de leurs performances et faire des propositions prescriptives. 


\section{Bibliographie}

BARTOL, A. (1990), Communication et organisation, Paris, Éditions d'Organisation, 175 pages.

Bartoli, A. et P. Hermel (1989), Le développement de l'entreprise, Paris, Économica, 421 pages.

De BANVILle, E. et J. VérIlhaC (1983), « Le capital redistribué », Saint-Étienne, CRESAL, 181 pages.

Chappoz, Y. et C. Thölot (1991), «Guide de l'action économique en Rhône-Alpes », Lyon, IRES, 198 pages.

Fuion, L.J. (1991), Vision et relations : clés du succès de l'entrepreneur, Montréal, Éditions de l'entrepreneur, 272 pages.

GrefFe, X. (1989), Décentraliser pour l'emploi, Paris, Économica, 297 pages.

HERMEL, P. (1989), Qualité et management stratégique, Paris, Éditions d'Organisation, 160 pages.

Julien, P.A. et M. Marchesnay (1988), La petite entreprise. Principes d'économie et de gestion, Paris, Vuibert, 288 pages.

LORRAIN, J. et L. Dussault (1988), « Les entrepreneurs artisans et opportunistes : une comparaison de leurs comportements de gestion », Revue Internationale PME, vol. $1, \mathrm{n}^{\circ} 2$, p. $157-176$.

MaIllat, D. (1988), «La revitalisation des régions industrielles : le rôle des PME et du milieu », Revue Internationale PME, vol. 1, $\mathrm{n}^{\circ} 1, \mathrm{p} .7-22$.

MaRchesnay, M. (1986), La stratégie. Du diagnostic à la décision industrielle, Paris, Chotard, 248 pages.

MARChESNAY, M. (1988a), «PME : quelle " techno-logique"? », Revue Internationale $P M E$, vol. $1, \mathrm{n}^{\circ} 2$, p. 223-240.

MARCHESNAY, M. (1988b), «La mercatique de la petite entreprise », Revue Internationale $P M E$, vol. 1 , nos $3-4$, p. 259-276.

MARCHESNAY, M. (1988c), "De la veille technologique au pilotage stratégique », Revue Internationale PME, vol. $1, \mathrm{n}^{\text {os }} 3-4$, p. 335-340.

Marchesnay, M. (1991a), Économie d'entreprise, Paris, Eyrolles, 110 pages.

MARChESNAY, M. (1991b), « La transaction, outil d'analyse stratégique ? », dans Identités de la gestion. Mélanges en l'honneur du Professeur Lassègue, Paris, Vuibert, p. $135-146$.

PACHE, G. (1991), «L'impact des stratégies d'entreprises sur l'organisation industrielle : PME et réseaux de compétences ", Revue d'économie industrielle $\mathrm{n}^{\circ} 56, \mathrm{p}$. 58-70.

Pecqueur, B. (1989), Le développement local, Paris, Syros, 148 pages.

PorTer, M. (1990), De l'avantage concurrentiel à la stratégie d'entreprise », HavardL'expansion, $\mathrm{n}^{\circ} 55$, p. 6-25.

PoRTER, M. (1991), «Enquête sur la concurrence des nations », Havard-L'expansion, $\mathrm{n}^{\circ} 59$, p. 13-33.

Rousseau, M. (1989), Le management des économies locales, Paris, Eyrolles, 166 pages. 